\title{
Dinámica folicular de vaquillas Criollas al pastoreo en el altiplano ecuatoriano
}

\author{
Ayala, L.E.' ; Pesantez, J.L'; Rodas, E.R. ${ }^{\text {; }}$; Dután, J.B. ${ }^{\text {; }}$ Calle, G.R. ${ }^{\text {; }}$; Murillo, Y.A. ${ }^{\text {; }}$ Vázquez, J.M. ${ }^{\text {; }}$ Nieto, P.E. ${ }^{1}$; Ortega, V.V. ${ }^{2}$ \\ y Samaniego, J.X. ${ }^{2}$
}

'Facultad de Ciencias Agropecuarias; Universidad de Cuenca. Campus Yanuncay. Cuenca. Ecuador.

${ }^{2}$ Médico Veterinario asociado.

PALABRAS CLAVE ADICIONALES

Dinámica Folicular.

Criollas.

Vaquillas.

\section{RESUMEN}

El objetivo fue caracterizar el parrón de comportamiento de la dinámica folicular en vaquillas Criollas (CR) al pastoreo en la sierra alta del Ecuador. Se realizó en la granja experimental Irquis de la Universidad

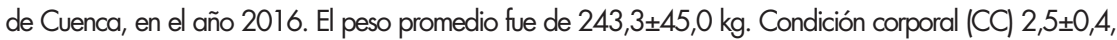
escala $1-5$ puntos. Edad 19,9 $\pm 4,8$ meses. Se realizaron ecografías diarias durante un ciclo estral. Cada $48 \mathrm{~h}$ se determinó los niveles de Progesterona (P4). Se estableció un patrón de dos y tres ondas foliculares (44,4 y $55,6 \%$, respectivamente). El promedio de duración del ciclo estral fue de $20,3 \pm 0,03$ días (dos ondas) y de $23,6 \pm 0,02$ días (tres ondas). El tamaño del folículo preovulatorio (FPO) fue de $15,3 \pm 0,04 \mathrm{~mm}$ para animales de dos ondas y para las de tres ondas $13,8 \pm 1,48 \mathrm{~mm}$. El folículo subordinado (FS) alcanzó su máximo tamaño el día $4,0 \pm 0,04$, con $8,0 \pm 0,04 \mathrm{~mm}$ (dos ondas) y 4,8 $\pm 0,03$ días con $7,4 \pm 0,03 \mathrm{~mm}$ (tres ondas). El desarrollo del cuerpo lúteo (CL) presentó tres fases: crecimiento (hasta el día 6), estática (6-18 días) y regresión (>18 días).

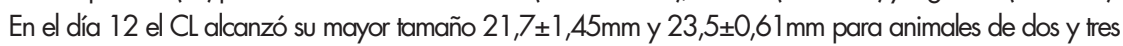
ondas, respectivamente. La P4 alcanzó niveles superiores a $\mathrm{lng} / \mathrm{ml}$ a partir del día 4 (5,8 8 3,35ng/ml dos ondas y $5,1 \pm 1,15 \mathrm{ng} / \mathrm{ml}$ tres ondas). Los niveles de $\mathrm{P} 4$ durante el ciclo estral fueron más altos que los reportados en otras razas. En conclusión, las vaquillas de genotipo Criollo poseen características propias, las cuales se ven influenciadas por el patrón de comportamiento folicular (dos o tres ondas).

\section{Follicular Dynamics of Creole heifers under grazing conditions in the Ecuadorian highlands}

\section{SUMMARY}

\section{ADDITIONAL KEYWORDS}

Follicular dinamice.

Creoles.

Heifers.

INFORMATION

Cronología del artículo.

Recibido/Received: 05.12.2017

Aceptado/Accepted: 02.06.2018

On-line: 07.04.2019

Correspondencia a los autores/Contact e-mail:

jorgereivax@hotmail.com

\section{INTRODUCCIÓN}

El bovino criollo americano es descendiente del ganado originario de la península Ibérica en la época de la conquista (Primo 1992, p. 421) que adaptó su constitución y aptitudes productivas a las variadas condiciones ambientales de la región. Las cuales a través del tiempo mediante selección natural en las diferentes regiones agroecológicas que ocuparon, permitieron que estos bovinos adquirieran características impor- tantes como rusticidad, adaptabilidad y resistencia a las enfermedades (Mendoza et al. 2002, p. 218; Aguirre et al. 2011, p. 393). Sin embargo, por ser consideradas poco productivas, estas poblaciones de animales denominadas Criollas paulatinamente han sido sustituidas o absorbidas por razas especializadas como Holstein y Brown Swiss (Zárate et al. 2010, p. 122). Si bien los animales de razas lecheras han sido mejorados genéticamente para producir grandes cantidades de leche; también, es cierto que suelen presentar problemas de 
fertilidad y mayor riesgo de contraer enfermedades. Por el contrario; algunas razas consideradas como Criollas en Ecuador (Negro Lojano, Encerado y Pintado o Cajamarca) tienen cualidades notables, como su alto instinto materno, excepcional resistencia a las condiciones ambientales adversas, elevada capacidad para aprovechar la escasa, dispersa y variada vegetación, como rastrojos de los cultivos y una mayor habilidad de producir y reproducirse sin la tecnificación apropiada (Aguirre et al. 2011, p. 392).

Las características reproductivas útiles para mejorar los rebaños mediante el uso de las tecnologías de fertilidad asistida no han sido estudiadas en el ganado Criollo; ejemplo de ello es el desconocimiento sobre la dinámica folicular, número de folículos reclutados por onda, concentraciones de hormonas esteroideas, entre otras; lo cual dificulta la aplicación exitosa de tratamientos para mejorar la eficiencia reproductiva (Comizzoli et al. 2000, p. 495; Andrabi \& Maxwell 2007, p. 228). La dinámica folicular ha sido descrita a detalle en Bos taurus y Bos indicus (Bo et al. 2003, p. 307-326). En los últimos años a nivel mundial se viene estudiando el comportamiento de la dinámica folicular en ganado denominado Criollo con la finalidad de crear bancos de material genético de estas razas en peligro de extinción (García \& García 1998 , p. 377). Chasombat et al. (2013, p. 21), determinaron que el desarrollo folicular del ganado Criollo tailandés tenía un comportamiento similar al B. taurus y B. indicus; sin embargo, presentaron características propias que difieren de las razas europeas. En Colombia, algunas razas de ganado Criollo muestran un alto grado de adaptación a las condiciones medioambientales del trópico, en términos de su buen desempeño reproductivo (Báez et al. 2007, p. online). En estas razas se han logrado describir algunas de las variables fisiológicas particulares, como los niveles basales de progesterona durante la fase folicular y la dinámica de cambio durante la fase luteal (Grajales \& Hernández 2008, p. online), lo cual ha contribuido a conocer las particularidades fisiológicas de este grupo genético y mejorar su eficiencia reproductiva. Estos hallazgos estimulan a avanzar en este tipo de investigación básica, con lo cual se dispondría de mayores elementos de juicio para identificar, analizar, comprender o explicar los mecanismos fisiológicos de adaptación; lamentablemente, en la actualidad en el Ecuador no se cuenta con información en los aspectos reproductivos antes mencionados en el ganado de genotipo Criollo local. Por lo tanto, se planteó como objetivo caracterizar la dinámica folicular de vaquillas Criollas pastoreadas en el altiplano ecuatoriano.

\section{MATERIALES Y MÉTODOS}

La investigación se llevó a cabo en la granja experimental "Irquis", perteneciente a la Facultad de Ciencias Agropecuarias, de la Universidad de Cuenca, al sur del Ecuador. Situada en las coordenadas geográficas -3.080212 y -79.075422 , a una altura de $2650 \mathrm{msnm}$, temperatura promedio de $14^{\circ} \mathrm{C}$, humedad relativa $80 \%$ y pluviosidad entre $800 \mathrm{~mm}$ y $2.000 \mathrm{~mm}$ anuales. Se tuvo presente la normativa del código sanitario para animales terrestres, capítulo 7.8 "Utilización de animales en la investigación y educación”, de la Organización Mundial de Sanidad Animal (OIE 2017, p. 01-12).

\section{ANIMALES}

Se analizaron 9 vaquillas de genotipo Criollo (CR), sometidas a los tratamientos una vez que alcanzaron la pubertad (celo detectado y presencia de cuerpo lúteo por ultrasonografía (US)). Los animales permanecieron en las instalaciones durante el año 2016, sometidas a las mismas condiciones de manejo, sanidad, alimentadas a base de pasturas (kikuyo, raygrass, trébol rojo y blanco), suplementadas con heno y sales minerales. El peso promedio fue de $243,3 \pm 45,0 \mathrm{~kg}$, condición corporal

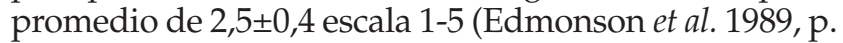
69) y edad de $19,9 \pm 4,8$ meses.

\section{DISEÑO EXPERIMENTAL}

En el presente trabajo se describió la dinámica folicular durante un ciclo estral y dentro de él se determinó el patrón de comportamiento en vaquillas con dos y tres ondas foliculares.

\section{PROTOCOLO DE SINCRONIZACIÓN DEL ESTRO}

El ciclo estral de las vaquillas fue evaluado después de la sincronización del estro. Para ello se administró dos dosis de $25 \mathrm{mg}$ de PGF2 $\alpha$ (Lutalise ${ }^{\circledR}$, Zoetis) por vía intramuscular (IM), aplicadas a un intervalo de 11 días. Luego de la última dosis de PGF2 $\alpha$, se colocó un parche adhesivo en la unión sacro-coxígea para la detección de celo, la cual se realizó por observación visual durante un periodo de 30 minutos cada 8 a 12 horas. Se consideró que la vaca estaba en celo cuando se dejó montar por otra y existió un cambió de coloración del parche.

\section{EVALUACIÓN ULTRASONOGRÁFICA}

Para el estudio de la dinámica folicular se realizó un chequeo ecográfico transrectal con un transductor linear (Prosound $2^{\circledR}$, Aloka, 7,5 Mhz). En cada animal se evaluaron los dos ovarios por la técnica de barrido en sentido latero-medial, dorso-ventral y cráneo-caudal, primero el derecho y luego el izquierdo, tal como lo describió Perea et al. (1998, p. 16). La valoración ultrasonográfica se realizó cada 24 horas a partir del día 0 (día de inicio del celo) hasta la siguiente ovulación. Se midieron los folículos $>3 \mathrm{~mm}$ y se dibujó la posición relativa de cada uno de ellos en un registro de campo con el fin de hacer mapeos ováricos, evaluar el número, diámetro de los folículos y del cuerpo lúteo (CL), como lo describió Ojeda et al. (2014, p. 4133).

\section{MuestRAS DE SANGRE}

Las muestras $(10 \mathrm{ml})$ se obtuvieron cada dos días mediante punción de la vena yugular, a partir del día cero (día del celo) hasta el final del ciclo estral, se utilizó tubos Vacutainer ${ }^{\mathrm{TM}}$ heparinizados y agujas 16GX1,5", específicas para estos tubos. Inmediatamente las muestras fueron centrifugadas a $2.500 \mathrm{rpm}$ durante $15 \mathrm{~min}$., luego se tomó el sobrenadante $(2 \mathrm{ml})$ de cada muestra y se colocó en un vial que se mantuvo en congelación $\left(-20^{\circ} \mathrm{C}\right)$ hasta su análisis. La determinación de la concentración de progesterona se realizó mediante test inmunológico in vitro con el método de electroquimioluminiscencia utilizando plasma heparinizado. El reactivo utilizado fue Progesterone II (Cobas $\left.{ }^{\circledR}\right)$.

Con respecto a la dinámica folicular se definieron las siguientes variables: 
“Onda folicular", se consideró retrospectivamente a partir del tamaño máximo de un folículo dominante y el momento en que este folículo inició su desarrollo junto con los demás de su cohorte.

"Número de ondas foliculares", sumatoria de eventos de los grupos de folículos que cumplieron los eventos de reclutamiento, selección, dominancia, atresia u ovulación (Kanitz 2003, p. 188).

"Día de emergencia de la onda folicular", día en el cual aparecieron nuevos folículos con diámetros $>3 \mathrm{~mm}$.

"Número de folículos reclutados" total de folículos de una cohorte que inició su crecimiento en cada onda.

"Folículo dominante", folículo que alcanzó el máximo tamaño y predominó sobre los demás de su cohorte durante una onda folicular.

"Día en el cual el folículo alcanzó la etapa de dominancia", día en el cual un solo folículo continuó creciendo, mientras que los subordinados dejaron de crecer e iniciaron su atresia; el diámetro que alcanzó el folículo seleccionado en este día fue considerado como "el diámetro con el cual alcanza la dominancia folicular el FD (Evans et al. 2000, p. 701).

"Diámetro máximo del folículo dominante", tamaño máximo que alcanzó el folículo dominante durante una onda de crecimiento".

"Tasa de crecimiento de los folículos dominante y subordinado (mm/día)", se determinó restando a la talla máxima, la talla inicial del folículo, dividido entre los días transcurridos entre ambos estadios (Savio et al. 1988, p. 664).

Con respecto al cuerpo lúteo se determinaron las siguientes variables:

"Diámetro del CL", a través de las medidas de ancho y largo del CL.

"Día de aparición del cuerpo lúteo", al primer día en el cual fue observado mediante ecografía esta estructura.

"Fase de crecimiento, estática y regresión del cuerpo lúteo", fue considerada desde su aparición hasta cuando alcanzó su máximo diámetro. La fase de estática fue determinada por el cese del crecimiento del CL, seguida de la disminución del tamaño de esta estructura (fase de regresión).

"Día inicial de funcionalidad del cuerpo lúteo", fue determinado como el día en el que se detectó una concentración de progesterona $\geq 1 \mathrm{ng} / \mathrm{ml}$.

\section{ANÁLISIS ESTADÍSTICO}

Para el análisis estadístico se aplicó un modelo lineal mixto a través del procedimiento MIXED del SAS (2013) vw 9.3; dónde, se utilizó la metodología de máxima verosimilitud restringida (REML) para estudiar los efectos del número de ondas foliculares y animal en los tratamientos (SAS, 2013). El procedimiento Satterthwaite (DDFM=SATTERTH) en el modelo para obtener los grados de libertad correctos para la tabla de prueba de efectos fijos.
El modelo matemático utilizado fue: Yijk = Ti + $\mathrm{Aj}(\mathrm{Ti})+$ Eijk

Donde:

Yijk= Variable respuesta

$\mathrm{Ti}=$ Efecto fijo del i-ésimo ondas foliculares (2,3..)

$\mathrm{Aj}(\mathrm{Ti})=$ Efecto aleatorio de la j-ésimo animal anidado en el i-ésimo tratamiento.

Eijk = Error aleatorio debido a cada observación NID (0, s2e).

Se aplicó la dócima de Tukey-Kramer (1956, p. 307) para la comparación múltiple de las medias de los mínimos cuadrados.

\section{RESULTADOS Y DISCUSIÓN}

\section{ONDAS FOLICULARES}

En total se observaron nueve ciclos estrales, de los cuales cinco presentaron un patrón de tres ondas de crecimiento folicular (55,6\%) Figura 1A y cuatro dos ondas (44,4\%) Figura 1B. Perry et al. (1991, p. 2551); Wolfenson et al. (2004, p. 1050) determinaron que el tipo de manejo, características raciales y probablemente otros factores como la alimentación, condiciones ambientales, entre otros, influyen en la dinámica folicular. Esto podría explicar la variabilidad en el número de ondas determinadas en diferentes trabajos en los últimos años, como el realizado por Sirois \& Fortune (1988, p. 312) en vaquillas lecheras (Holstein), quienes establecieron que un $70 \%$ presentaron tres ondas, $20 \%$ dos y $10 \%$ una onda folicular; es decir, se mantiene un mayor porcentaje de animales con tres ondas foliculares. Igual tendencia obtuvo Noseir et al. (2003, p. 5) al valorar vaquillas nativas quienes describieron que el 71,4\% presentaron una actividad folicular de 3 ondas. Sin embargo, en contraposición Chasombat et al. (2013, p. 17), establecieron que las vaquillas nativas tailandesas presentaban dos $(70 \%)$ y tres $(30 \%)$ ondas. Se considera que esta diferencia puede ser explicada por la tendencia cárnica de estas vaquillas a diferencia de los animales del estudio que tienen aptitud lechera. Igual porcentaje se obtuvo en ganado criollo mexicano de carne $77,3 \%$ dos ondas y 22,7\% tres ondas (Quezada et al. 2014, p. 678).

\section{DURACIÓN DEL CICLO ESTRAL:}

El promedio de duración del ciclo estral, en vacas con patrón folicular de dos ondas fue de 20,3 $\pm 0,03$ días y de tres ondas 23,6 $\pm 0,02$ días. Estos valores se encuentran dentro de los límites biológicos determinados por varios autores para vaquillas Criollas de dos $\mathrm{y}$ tres ondas, quienes manifiestan que los animales de dos ondas presentan menor longitud del ciclo estral en comparación con las de tres, con valores entre 19,8 y 22,5 días, respectivamente (Noseir 2003, p. 02; Chasombat et al. 2013, p. 18). Sin embargo, autores como Denis et al. (2007, p. 25), en trabajos realizados en Cuba sobre vacas cruce Holstein 5/8 y Cebú 3/8 (Siboney) semiestabuladas, establecieron que las de dos ondas tienen un intervalo interovulatorio de $18,38 \pm 0,67$ y las de tres $20,85 \pm 0,31$, si bien las vacas de tres ondas mantienen 

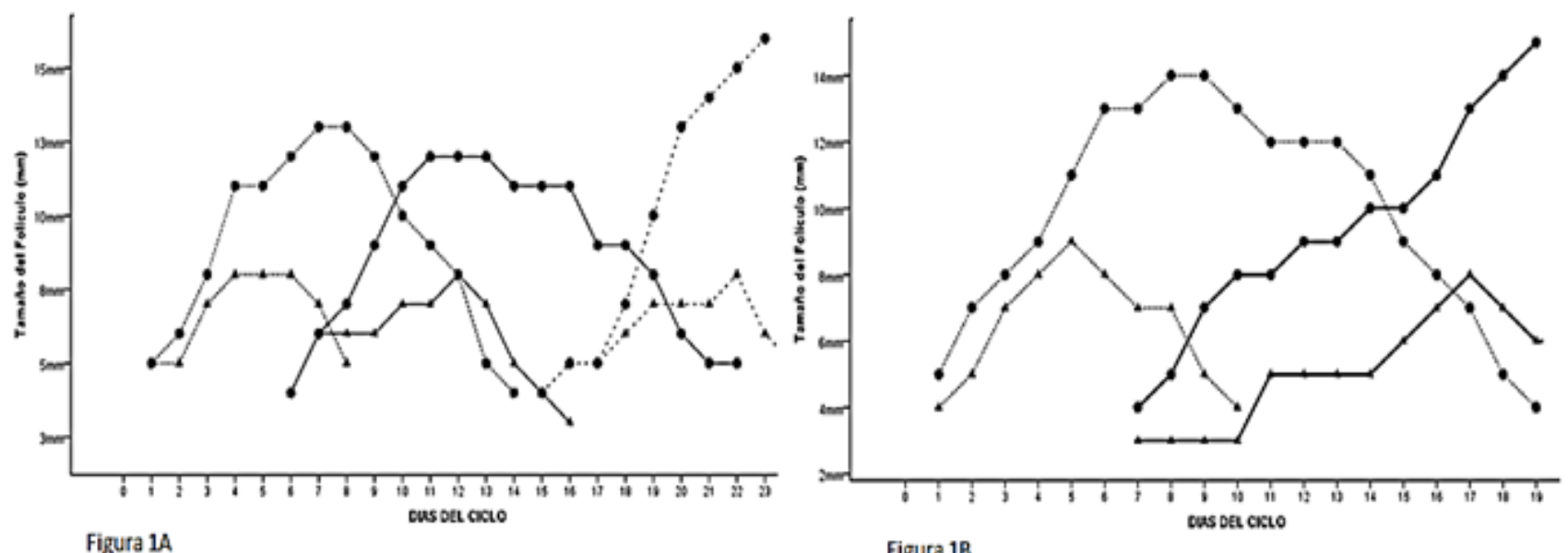

Figura 1. (A) Dinámica folicular en vaquilla criolla (\#288) de tres ondas, (B) Dinámica folicular en vaquilla criolla (\#9843) de dos ondas (A) Follicular Dynamics in Creole heifer (\#288) of three waves, (B) follicular Dynamics in Creole heifer (\#9843) of two waves). Folículo dominante de $1^{\mathrm{a}}$ onda $(\bullet \ldots \bullet)$; folículo dominante de $2^{\mathrm{a}}$ onda folicular $(\bullet \bullet \bullet)$; folículo ovulatorio (•- - -

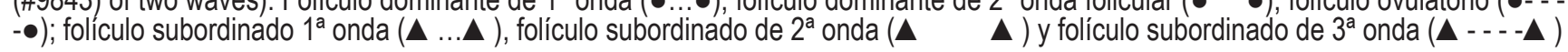

un período más largo frente a las de dos, los valores obtenidos son menores, seguramente como consecuencia del ambiente, sistema de manejo y el cruce de esta raza, que pudo afectar el comportamiento fisiológico ovárico entre razas de Bos taurus y B. indicus (Henao 2010, p. 5583).

\section{DINÁMICA FOLICULAR}

La caracterización de la dinámica folicular en las vaquillas de dos y tres ondas se detalla en la Tabla I. Los folículos fueron registrados a partir de $3 \mathrm{~mm}$. La primera onda emergió el día uno tanto para las vaquillas de dos y tres ondas, con una duración de 7 y 6 días, respectivamente. La segunda onda inició el día $7,8 \pm 1,60$ para animales de dos ondas y el día $6,6 \pm 0,05$ para los de tres ondas $(\mathrm{P}<0,05)$, con una duración de 13 y 7 días (dos y tres ondas, respectivamente). La tercera onda surgió el día $13,2 \pm 1,30$, con una duración de 10 días. Por los datos obtenidos se puede inferir que la onda ovulatoria en vaquillas de tres ondas (tercera onda) es más corta (10 días), comparada con la onda ovulatoria de las vaquillas de dos ondas (13 días), tendencia similar obtuvo Quezada et al. (2014, p. 678), valorando vaquillas criollas mexicanas y Chasombat et al. (2013, p. 18) en vaquillas tailandesas.

Díaz $(1999$, p. 6) señaló que cada onda folicular se caracteriza por la emergencia simultánea de un grupo de 5 a 7 folículos $<5 \mathrm{~mm}$ de diámetro. En el presente trabajo el número de folículos reclutados fue mayor (911) en los animales de tres ondas $(P<0,05)$ comparado con las vaquillas de dos (5 y 6) (Tabla I).

Tabla I. Media y error estándar del patrón de comportamiento del folículo dominante (FD) y subordinado (FS) en la primera, segunda y tercera onda de las vaquillas de genotipo Criollo con dos y tres ondas foliculares (Mean and standard error of the dominant follicle (FD) and subordinate (FS) behavior pattern in the first, second and third wave of heifers of Creole genotype con two and three follicular waves).

\begin{tabular}{|c|c|c|c|c|c|c|}
\hline & \multicolumn{2}{|c|}{ Primera onda } & \multicolumn{2}{|c|}{ Segunda onda } & \multicolumn{2}{|c|}{ Tercera onda } \\
\hline & Dos ondas & Tres ondas & Dos ondas & Tres ondas & Dos ondas & Tres ondas \\
\hline \multicolumn{7}{|l|}{ Folículo dominante: } \\
\hline Día de emergencia de la onda & 1 & 1 & $7,8 \pm 1,60$ & $6,6 \pm 0,05^{*}$ & & $13,2 \pm 1,30$ \\
\hline$N^{\circ}$ de folículos reclutados & $6,6 \pm 1,46$ & $9,9 \pm 1,54$ & $5,0 \pm 0,22$ & $10,2 \pm 0,14^{*}$ & & $11,4 \pm 4,39$ \\
\hline Día en el cual alcanza la dominancia & $4,0 \pm 0,04$ & $4,8 \pm 0,03^{*}$ & $14,5 \pm 0,07$ & $12,0 \pm 0,07^{*}$ & & $18,8 \pm 1,09$ \\
\hline Diámetro con el cual alcanza dominancia (mm) & $9,3 \pm 0,09$ & $11,0 \pm 0,05^{*}$ & $10,0 \pm 0,09$ & $9,6 \pm 0,09$ & & $9,8 \pm 1,30$ \\
\hline Día en el cual alcanza el diámetro máximo FD & $7,8 \pm 0,07$ & $7,4 \pm 0,06^{*}$ & $19,3 \pm 0,06$ & $12,4 \pm 0,07^{*}$ & & $23,0 \pm 0,71$ \\
\hline Diámetro máximo FD (mm) & $13,2 \pm 2,2$ & $12,2 \pm 1,71$ & $15,3 \pm 0,04$ & $10,2 \pm 0,04^{*}$ & & $13,8 \pm 1,48$ \\
\hline Tasa de crecimiento (mm/día) & $1,0 \pm 0,12$ & $1,2 \pm 0,09$ & $0,9 \pm 0,13$ & $1,1 \pm 0,10$ & & $1,1 \pm 0,27$ \\
\hline \multicolumn{7}{|l|}{ Folículo subordinado: } \\
\hline Diámetro máximo (mm) & $8,0 \pm 0,04$ & $7,4 \pm 0,03^{*}$ & $7,1 \pm 1,46$ & $7,8 \pm 1,31$ & & $7,8 \pm 0,84$ \\
\hline Día de diámetro máximo & $4,0 \pm 0,04$ & $4,8 \pm 0,03^{*}$ & $14,5 \pm 0,07$ & $12,0 \pm 0,07^{*}$ & & $18,8 \pm 1,09$ \\
\hline Tasa de crecimiento (mm/día) & $0,9 \pm 0,18$ & $0,9 \pm 0,15$ & $0,5 \pm 0,13$ & $0,7 \pm 0,09^{*}$ & & $0,7 \pm 0,17$ \\
\hline
\end{tabular}




\section{FOLÍCULO DOMINANTE (FD)}

El día en el cual el folículo alcanzó la dominancia (FD) en la primera onda, fue mayor para las vaquillas de tres ondas $(\mathrm{P}<0,05)$ en comparación con las vaquillas de dos $(4,8 \pm 0,03$ y 4,0 0 0,04 días, respectivamente). Perea et al. (1998, p. 20), establecieron un patrón de comportamiento diferente en vaquillas mestizas Bos taurus (Holstein y Pardo suizo), que fue menor en animales de dos ondas (3,4 $\pm 1,9$ días).

El diámetro con el cual el folículo alcanza la dominancia (FD) en la primera onda fue de mayor $(11,0 \pm 0,05 \mathrm{~mm})$ en los animales de tres ondas $(\mathrm{P}<0,05)$. Sin embargo, en la segunda onda tanto las vaquillas de dos y tres ondas presentaron diámetros similares al alcanzar dominancia (P>0,05). Tabla I. Estos valores son corroborados por trabajos realizados en vaquillas mestizas de Venezuela por Perea et al. (1998, p. 20).

Al analizar el día en el cual el FD alcanza su diámetro máximo se estableció que en la primera y segunda onda las vaquillas de tres ondas necesitaban menos días, que las de dos ondas $(\mathrm{P}<0,05)$.

Así mismo, el diámetro máximo que alcanzó el FD en la segunda onda fue mayor en las vaquillas de dos ondas, seguramente debido a su proximidad a la ovulación.

La tasa de crecimiento del FD fue similar $(\mathrm{P}>0,05)$ en los dos grupos de vaquillas en estudio (dos y tres ondas), con valores entre 0,9 y 1,2mm/día, datos similares de crecimiento folicular diario $(1,2 \pm 0,08 \mathrm{~mm} /$ día $)$ obtuvo Sartori et al. (2004, p. 913) al valorar a vaquillas Holstein en USA.

\section{FOLÍCULO PREOVULATORIO (FPO)}

Sartori et al. (2004, p. 913) establecieron que el tamaño máximo del folículo preovulatorio (FPO) en vaquillas Holstein fue de $(15,2 \pm 0,3 \mathrm{~mm})$. Sin embargo, Echternkamp et al. (2014, p. 3560) determinaron un folículo preovulatorio de 13,9 $90,5 \mathrm{~mm}$ de diámetro en vaquillas Holstein de 2 años. En las vaquillas Criollas de la presente investigación se fijó un valor de FPO $15,3 \pm 0,04 \mathrm{~mm}$ para las de dos ondas y $13,8 \pm 1,48 \mathrm{~mm}$ para animales de tres ondas, valores que se encuentran dentro de los parámetros establecidos por los trabajos antes citados al evaluar el folículo preovulatorio. En contraposición, Chasombat et al. (2013, p. 18); Quezada et al. (2014, p. 680), determinaron un promedio del tamaño del FPO inferior que fluctúa entre 8 y 10mm, en vaquillas criollas posiblemente por la tendencia cárnica de los animales analizados, esto basado en el criterio emitido por Bó et al. (2003, p. 310) quienes describen que el Bos indicus tiene FPO más pequeños que el Bos taurus (10-12mm y $14-20 \mathrm{~mm}$, respectivamente).

\section{FolícULO SUBORDINADO (FS)}

La descripción del FS en cada una de las ondas se detalla en la Tabla I. El FS de la primera onda al-

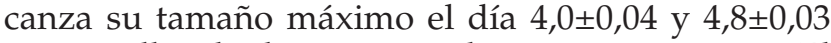
en vaquillas de dos y tres ondas, respectivamente. El tamaño máximo alcanzado por el FS en la primera onda fue de $8,0 \pm 0,04$ y 7,4 $40,03 \mathrm{~mm}$ para las de dos y tres ondas, respectivamente $(\mathrm{P}<0,05)$. Chasombat et al. (2013, p.19) al estudiar vaquillas nativas tailandesas determinaron que el FS en la primera onda alcanzó su máximo tamaño en días similares al del presente estudio $(5,49 \pm 0,16$ vaquillas de dos ondas y 5,25 $\pm 0,11$ tres ondas). Sin embargo, el tamaño máximo alcanzado por el FS fue la mitad al comparar con el presente trabajo $(4,1 \pm 0,12$ y $4,1 \pm 0,04 \mathrm{~mm})$, seguramente este comportamiento está ligado al tamaño final de los folículos en esta raza.

En la segunda onda folicular el FS del grupo de animales de tres ondas alcanza su máximo tamaño dos días antes que las del grupo de dos ondas $(14,5 \pm 0,07$ y $12,0 \pm 0,07$ días), con diferencia entre grupos $(\mathrm{P}<0,05)$. El tamaño máximo del FS es similar entre animales de dos y tres ondas $(7,1 \pm 1,46$ y $7,8 \pm 1,31 \mathrm{~mm}$, respectivamente). El FS en la tercera onda alcanzó un tamaño máximo de $7,8 \pm 0,84 \mathrm{~mm}$ el día $18,8 \pm 1,09$.

La tasa de crecimiento del FS fluctúa entre $0,5 \pm 0,13$ y $0,9 \pm 0,18 \mathrm{~mm} /$ día. Presenta un patrón de crecimiento similar en la primera onda y difiere en la segunda onda $(0,5 \pm 0,13$ y $0,7 \pm 0,09 \mathrm{~mm} /$ día) para las vaquillas de dos $\mathrm{y}$ tres ondas, respectivamente.

\section{CuerPo lúteO}

El desarrollo del CL en la presente investigación presentó tres fases: crecimiento (hasta día 6), estática (6-18) y regresión (>18), lo que concuerda con lo expresado por Chasombat et al. (2013, p. 20). El cuerpo lúteo apareció más temprano (3,1 10,09 días) en vaquillas de dos ondas $(\mathrm{P}<0,05)$, comparado con las de tres ondas $(4,0 \pm 0,05)$. Denis et al. (2007, p. 28), establecieron un promedio similar 3,0 0 , 48 en vacas Siboney. Datos semejantes fueron reportados por Perea et al. (1998, p. 21) quienes describieron la primera observación del CL en novillas mestizas el día $4,0 \pm 1,9$.

El día cuatro del ciclo estral las vaquillas de dos y tres ondas presentan un tamaño de CL similar $(14,4 \pm 0,23 \mathrm{~mm}$ y $13,6 \pm 0,31 \mathrm{~mm}$, respectivamente), Tabla II. En el día 12 el CL alcanzó su tamaño máximo tanto en las vaquillas de dos como en las de tres ondas

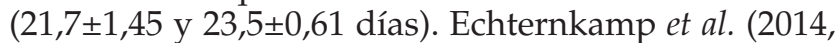
p. 3561) establecieron promedios similares en cuanto al tamaño máximo del CL (20,8 $80,4 \mathrm{~mm}$ día 12$)$ en vaquillas Holstein; sin embargo, otros investigadores como Perea et al. (1998, p. 21); Chasombat et al. (2013, p. 20); Chacón et al. (2005, p.108) determinaron valores inferiores $(1.9 \pm 0,3 \mathrm{~cm}$ y $15,14 \pm 0,14 \mathrm{~mm}$, respectivamente), esta diferencia en el tamaño del CL pudo ser debido al efecto raza.

La tasa de crecimiento fue similar para los dos grupos $(\mathrm{P}>0,05)$ presentando promedios de $1,4 \pm 0,30 \mathrm{~mm} /$ día y $1,9 \pm 0,18 \mathrm{~mm} /$ día para las vaquillas de dos y tres ondas, respectivamente.

La fase de regresión del CL inició el día 18 del ciclo estral en vaquillas de dos ondas con un tamaño promedio de $16,3 \pm 1,25 \mathrm{~mm}$ y en las vaquillas de tres ondas esta fase se presentó el día 20, con un tamaño de CL de 16,6 $\pm 1,14 \mathrm{~mm}$ (Tabla II).

\section{Progesterona}

Los niveles de progesterona mayores a $1 \mathrm{ng} / \mathrm{ml}$ se presentaron a partir del cuarto día del ciclo estral tanto en vaquillas de dos $(5,8 \pm 3,35 \mathrm{ng} / \mathrm{ml})$ y tres ondas 
Tabla II. Media y error estándar del patrón de comportamiento del cuerpo lúteo (CL) y la progesterona en vaquillas de genotipo Criollo dos y tres ondas foliculares (Mean and standard error of the pattern of behavior of the body luteum (CL) and progesterone in heifers of Creole genotype two and three follicular waves).

\begin{tabular}{|c|c|c|c|c|c|}
\hline \multirow[b]{2}{*}{ Fase } & \multirow[b]{2}{*}{ Día } & \multicolumn{2}{|c|}{ Tamaño CL (mm) } & \multicolumn{2}{|c|}{ Progesterona (ng/ml) } \\
\hline & & Dos ondas & Tres ondas & Dos ondas & Tres ondas \\
\hline \multirow[t]{3}{*}{ Fase de crecimiento } & 0 & & & $0,7 \pm 0,18$ & $0,9 \pm 0,28$ \\
\hline & 2 & & & $0,7 \pm 0,14$ & $0,9 \pm 0,25$ \\
\hline & 4 & $14,4 \pm 0,23$ & $13,6 \pm 0,31$ & $5,8 \pm 3,35$ & $5,1 \pm 1,15$ \\
\hline \multirow[t]{6}{*}{ Fase de estática } & 6 & $17,6 \pm 1,12$ & $19,2 \pm 0,90$ & $10,5 \pm 3,01$ & $11,3 \pm 2,12$ \\
\hline & 8 & $19,63 \pm 1,06$ & $20,9 \pm 0,81$ & $20,6 \pm 5,47$ & $15,1 \pm 2,83$ \\
\hline & 10 & $20,8 \pm 1,29$ & $23,0 \pm 1,15$ & $19,6 \pm 3,84$ & $16,3 \pm 2,13$ \\
\hline & 12 & $21,7 \pm 1,45$ & $23,5 \pm 0,61$ & $16,5 \pm 3,56$ & $20,0 \pm 2,44$ \\
\hline & 14 & $21,6 \pm 1,31$ & $22,4 \pm 0,73$ & $16,3 \pm 3,23$ & $19,8 \pm 2,56$ \\
\hline & 16 & $18,3 \pm 1,10$ & $21,4 \pm 0,88$ & $12,0 \pm 4,98$ & $20,6 \pm 3,12$ \\
\hline \multirow[t]{3}{*}{ Fase de regresión } & 18 & $16,3 \pm 1,25$ & $19,7 \pm 1,15$ & $0,7 \pm 0,22$ & $13,7 \pm 2,34^{*}$ \\
\hline & 20 & $12,3 \pm 0,25$ & $16,6 \pm 1,14$ & $0,8 \pm 0,27$ & $4,8 \pm 2,30^{*}$ \\
\hline & 22 & & & & $0,9 \pm 0,16$ \\
\hline
\end{tabular}

${ }^{*}=$ diferencia estadística entre filas, prueba de Tukey-Kramer. $(P<0.05) ; C L=$ cuerpo lúteo; Día= del ciclo estral

$(5,1 \pm 1,15 \mathrm{ng} / \mathrm{ml})$. Estos niveles altos de $\mathrm{P}_{4}$ se mantienen en el caso de las vaquillas de dos ondas hasta el día $16(12,0 \pm 4,98 \mathrm{ng} / \mathrm{ml})$ y en las de tres ondas hasta el día $20(4,8 \pm 2,30 \mathrm{ng} / \mathrm{ml})$, a partir de estos días los niveles de $\mathrm{P}_{4}$ bajan a menos de $1 \mathrm{ng} / \mathrm{ml}$ (Tabla II). Es importante resaltar que los niveles de $\mathrm{P}_{4}$ en los animales de genotipo Criollo son altos llegando hasta valores de $20,6 \pm 5,47 \mathrm{ng} / \mathrm{ml}$ en vaquillas de dos ondas y $20,6 \pm 3,12 \mathrm{ng} / \mathrm{ml}$ en las de tres ondas. Esto podría ser una característica propia de este genotipo, ya que en trabajos realizados en vaquillas Holstein por Sartori et al. (2004, p. 913); Echternkamp et al. (2014, p. 3561) los valores más altos de progesterona no superan los $10 \mathrm{ng} / \mathrm{ml}$; así mismo, investigaciones realizadas en ganado criollo y mestizo por autores como Perea et al. (1998, p. 21); Chasombat et al. (2013, p. 21); Quezada et al. (2014, p. 681), los valores estuvieron por debajo de los $7 \mathrm{ng} / \mathrm{ml}$. Investigadores como Forde et al. (2009, p. 790); Gonella et al. (2010, p. 1978) señalaron que niveles altos de $\mathrm{P}_{4}$ mejoran el ambiente uterino y favorecen la implantación y el desarrollo embrionario. Por lo tanto, estos niveles elevados de $\mathrm{P}_{4}$ durante el ciclo estral en las vaquillas de genotipo Criollo podrían ser parte de la explicación de la alta fertilidad que presentan estos animales.

\section{CONCLUSIONES}

En conclusión, las vaquillas de genotipo Criollo criadas al pastoreo en el altiplano ecuatoriano, poseen características fisiológicas ováricas que se ven influenciadas por el patrón de comportamiento folicular (dos o tres ondas), propias de estos animales.

\section{BIBLIOGRAFÍA}

Aguirre, L, Bermeo, A, Maza, D \& Merino, L 2011, 'Estudio fenotipico y zoometrico del bovino Criollo de la sierra media y alta de la región sur del ecuador (RSE)', Actas Iberoamericanas de Conservación Animal, pp. 392-396, <https://www.google.com.ec/urlesa=t\&rct=i\&q=\&es $r c=s \&$ source $=$ web\&cd $=1$ \&cad $=$ rja\&uact $=8 \&$ ved $=0$ ahUKEw $\mid 19 \mathrm{NiW}$ jOLXAhVDj1QKHZRUBMsQFggkMAA\&url=hHtp\%3A\%2F\%2Fwww. uco.es\%2Fconbiand\%2Faica\%2Ftemplatemo_1 10_lin_photo\%2Fa rticulos\%2F2011\%2FAguirre2011_1_392_396.pdf\&usg=AOvV>.

Andrabi, S \& Maxwell, W 2007, 'A review on reproductive biotechnologies for conservation of endangered mammalian species', Animal reproduction science, vol 99, no. 3-4, pp. 223-243, <http://sci-hub. bz/10.1016/j.anireprosci.2006.07.002>.

Báez, G, Grajales, H \& Pérez, J 2007, 'Caracterización del ciclo estral mediante perfiles de esteroides (progesterona, $17 \beta$-estradiol) en la raza Costeño con cuernos (Bos taurus) en el trópico Colombiano', Livestock Research for Rural Development, vol 19, no. 132, <http:// www.lrrd.org//rrd19/9/baez19132.htm>.

Bó, G, Baruselli, P \& Martínez, M 2003, 'Pattern and manipulation of follicular development in Bos indicus cattle', Animal reproduction science, vol 78, no. 3-4, pp. 307-326, <http://sci-hub.bz/10.1016/ s0378-4320(03)00097-6>

Chacón, L, Vargas, M, Otero, R \& Villamil, A 2005, 'Seguimiento de la dinámica del ovario por ultrasonografia en novillas de raza Gir', Revista UDCA, vol 8, no. 2, pp. 103-110, <http://biblat.unam.mx/ es/revista/revista-udca-actualidad-divulgacion-cientifica/articulo/ seguimiento-de-la-dinamica-del-ovario-por-ultrasonografia-en-novillasde-la-raza-gir>.

Chasombat, J, Nagai, T, Parnpai, R \& Vongpralub, T 2013, 'Ovarian follicular dynamics and hormones throughout the estrous cycle in Thai native (Bos indicus) heifers', Animal Science Journal, vol 85, no. 1, pp. 15-24, <http://sci-hub.bz/10.1111/asj.12086>

Comizzoli, P, Mermillod, P \& Mauget, R 2000, 'Reproductive biotechnologies for endangered mammalian species', Reproduction Nutrition Development, vol 40, no. 5, pp. 493-504, <https://hal.archivesouvertes.fr/hal-00900403/document>.

Denis, R, Gil, A, Fuentes, D, Lliteras, E \& Chog, M 2007, 'Dinámica y sincronización de las ondas foliculares en vacas del genotipo Siboney de Cuba', Ciencia y Tecnología Ganadera, vol 1, no. 1-2, pp. 2332, <http://www.cima-minag.cu/wa_files/Vol_1_281-2_29Denis2. $\mathrm{pdf}$. 
Díaz, T 1999, 'Dinámica del desarrollo folicular ovárico durante el ciclo estral en el bovino', Revista Facultad de Ciencias Veterinarias UCV, vol 40, no. 1, pp. 3-18.

Echternkamp, S, Cushman, R \& Allan, M 2014, 'Size of ovulatory follicles in cattle expressing multiple ovulations naturally and its influence on corpus luteum development and fertility', Journal Animal Sciense, vol 87 , no. 11 , pp. 3556-3568, <https://www.animalsciencepublications.org/publications/jas/pdfs/87/1 1/3556>

Edmonson, A, Lean, I, Weaver, L, Farver, T \& Webster, G 1989, 'A body Condition Scoring Chart for Holstein Dairy Cows', Journal of Dairy Science, vol 72, no. 1, pp. 68-78, <http://sci-hub.bz/10.3168/ jds.s0022-0302(89)79081-0>.

Evans, A, Duffy, P, Hynes, N \& Boland, M 2000, 'Waves of follicle development during the estrous cycle in sheep', Theriogenology, vol 53, no. 3, pp. 699-715, <http://sci-hub.bz/10.1016/s0093$691 \times(99) 00268-x>$.

Forde, N, Carter, F, Fair, T, Crowe, M, Evans, A, Spencer, T, Bazer, F, McBride, R, Boland, M, O'Gaora, P, Lonergan, P \& Roche, J 2009, 'Progesterone-Regulated Changes in Endometrial Gene Expression Contribute to Advanced Conceptus Development in Cattle', Biology of Reproduction, vol 81, no. 4, pp. 784-794, <http://sci-hub. bz/10.1095/biolreprod.108.074336>.

García, B \& García, L 1998, 'Raza blanca Cacereña: Programa de conservación', Archivos de Zootecnia, vol 47, no. 178-179, pp. 371-380, <http://www.uco.es/organiza/servicios/publica/az/ php/img/web/05_13_09_45bartolom.pdfs.

Gonella, A, Grajales, H \& Hernández, A 2010, 'Ambiente receptivo uterino: control materno, control embrionario, muerte embrionaria', Revista MVZ. Córdoba, vol 15, no. 1, pp. 1976-7984, <http://www. scielo.org.co/pdf/mvz/v15n1/v15nlal 1.pdfs.

Grajales, H \& Hernández, A 2008, Niveles séricos de progesterona durante los días $0,5,10,15$ y 20 del ciclo estral en novillas Simmental x Cebú, Holstein x Cebú, Romosinuano y Cebú, bajo las condiciones del trópico cálido -húmedo Colombiano', Livestock Research for Rural Development, vol 20, no. 39, <http://www.Irrd.org/Irrd20/3/ graj20039.htm>.

Henao, G 2010, 'Algunos Factores Relacionados con la Dinámica Folicular en Bos indicus', Revista Facultad Nacional de Agronomía Medellín, vol 63, no. 2, pp. 5577-5586, <http://www.scielo.org. $\mathrm{co} / \mathrm{pdf} / \mathrm{rfnam} / \mathrm{v} 63 \mathrm{n} 2 / \mathrm{a} 11 \mathrm{v} 63 \mathrm{n} 01 . \mathrm{pdf}$.

Kanitz, W 2003, 'Follicular dynamic and ovulation in cattle- a review', Arch. Tierz, vol 46, no. 2, pp. 187-198, <http://archtierz.fbndummerstorf.de/pdf/2003/at03p187.pdf>.

Mendoza, M, Serrano, J, Ávila, R, García, M \& Méndez, N 2002, 'Caracterización morfométrica del Bovino Criollo mixteco', Archivos de Zootecnia, vol 51, no. 194, pp. 217-221, <https://dialnet.unirioja. es/descarga/articulo/279999.pdfs.

Noseir, W 2003, 'Ovarian follicular activity and hormonal profile during estrous cycle in cows: the development of 2 versus 3 waves', Reproductive Biology and Endocrinology, vol 1, no. 50, pp. 1-6, <htps:// sci-hub.bz/https://dx.doi.org/10.1 186\%2F1477-7827-1-50>.

Ojeda, A, Londoño, R, Gutierrez, C \& Gonella, A 2014, 'Dinámica folicular, crecimiento y regresión del cuerpo lúteo en búfalas multíparas y novillas', Revista MVZ Córdoba, vol 19, no. 2, pp. 4130-4140, <http://www.scielo.org.co/pdf/mvz/v19n2/v19n2a09.pdfs.
Organización mundial de sanidad animal 2017, Utilización de animales en la investigación y educación, viewed 27 Noviembre 2017, <http:// www.oie.int/fileadmin/Home/esp/Health_standards/tahc/current/ chapitre_aw_research_education.pdfs.

Perea, F, González, R, Cruz, R, Soto, E, Rincón, E, Gonzáles, C \& Villamediana, P 1998, 'Evaluación ultrasonográfica de la dinámica folicular en vacas y en novillas mestizas', Revista Científica , FCV-LUZ, vol 8, no. 1, pp. 14-24, <https://www.google.com.ec/urle sa=t\&rct=i\&q= \&esrc $=$ \&\&source $=$ web\&cd $=1$ \&cad $=r j a \&$ uact $=8 \&$ ved $=0$ ahUKEwiY 5JW_k-LXAhXJQyYKHR_CCCYQFggkMAA\&url=http\%3A\%2F\%2F www. produccioncientifica.luz.edu.ve\%2Findex. php\%2Fcientifica \%2Farticle\%2FviewFile\%2F 14304\%2F 14282\&usg=AOvVaw 1 jX>.

Perry, R, Corah, L, Kiracofe, G, Stevenson, J \& Beal, W 1991, 'Endocrine changes and ultrasonography of ovaries in suckled beef cows during resumption of postpartum estrous cycles', Journal of Animal Science, vol 69, no. 6, pp. 2548-2555, <https://www.animalsciencepublications.org/publications/jas/articles/69/6/2548>.

Primo, AT 1992, 'El ganado Bovino Iberico en las Americas: 500 años después', Archivos de zootecnia, vol 41, no. 154 (extra), pp. 421432,<https://dialnet.unirioja.es/descarga/articulo/278746.pdfs.

Quezada, A, Avendaño, L, Macías, U, Ramírez, J \& Correa, A 2014, 'Estrus behavior, ovarian dynamics, and progesterone secretion in Criollo cattle during estrous cycles with two and three follicular waves', Tropical Animal Health and Production, vol 46, no. 4, pp. 675-684, <http://sci-hub.bz/10.1007/s1 1250-014-0562-0>.

Sartori, R, Haughian, J, Shaver, R, Rosa, G \& Wiltbank, M 2004, 'Comparison of Ovarian Function and Circulating Steroids in Estrous Cycles of Holstein Heifers and Lactating Cows', Journal of Dairy Science, vol 87, no. 4, pp. 905-920, <http://sci-hub.bz/10.3168/ids. s0022-0302(04)73235-x>

Savio, J, Keenan, L, Boland, M \& Roche, J 1988, 'Pattern of growth of dominant follicles during the oestrus cycle of heifers', Journal of Reproduction \& Fertility, vol 83, no. 2, pp. 663-671, <http://www. reproduction-online.org/content/83/2/663.long>.

Sirois, J \& Fortune, J 1988, 'Ovarian Follicular Dynamics during the Estrous Cycle in Heifers Monitored by Real-Time Ultrasonography', Biology of Reproduction, vol 39, no. 2, pp. 308-317, <http://sci-hub. bz/10.1095/biolreprod39.2.308>

Tukey-Kramer, C. Y. (1956). Extension of multiple range tests to group means with Unequal numbers of replications. Biometrics, vol 12, no. 3, pp. 307-310, < http://www.jstor.org/stable/3001469? origin=JSTORpdf>.

Wolfenson, D, Inbar, G, Roth, Z, Kaim, M, Bloch, A \& Braw-Tal, R 2004, 'Follicular dynamics and concentrations of steroids and gonadotropins in lactating cows and nulliparous heifers', Theriogenology, vol 62, no. 6, pp. 1042-1055, <http://sci-hub.bz/10.1016/i.theriogenology.2003.12.020>.

Zárate, J, Ramírez, J \& Rodríguez, F 2010, 'Comportamiento reproductivo de vacas Criollas con amamantamiento restringido y sincronización del estro', Agronomía Mesoamericana, vol 21, no. 1, pp. 121-130. 